\title{
Third-harmonic generation in slow-light chalcogenide glass photonic crystal waveguides
}

\author{
Christelle Monat, ${ }^{1,2, *}$ Marcel Spurny, ${ }^{3}$ Christian Grillet, ${ }^{1}$ Liam O'Faolain, ${ }^{3}$ Thomas F. Krauss, ${ }^{3}$ \\ Benjamin J. Eggleton, ${ }^{1}$ Douglas Bulla, ${ }^{4}$ Steve Madden, ${ }^{4}$ and Barry Luther-Davies ${ }^{4}$ \\ ${ }^{1}$ Centre for Ultrahigh bandwidth Devices for Optical Systems (CUDOS), Institute of Photonics and Optical Science (IPOS), \\ School of Physics, University of Sydney, New South Wales 2006, Australia \\ ${ }^{2}$ Université de Lyon, Institut des Nanotechnologies de Lyon (INL)-UMR 5270, CNRS, Ecole Centrale de Lyon, Ecully, France \\ ${ }^{3}$ SUPA, School of Physics and Astronomy, University of St. Andrews, St. Andrews, Fife KY16 9SS, UK \\ ${ }^{4}$ CUDOS, Laser Physics Centre, The Australian National University, Canberra, Australian Capital Territory Australia \\ ${ }^{*}$ Corresponding author: christelle.monat@ec-lyon.fr
}

Received April 25, 2011; revised June 27, 2011; accepted June 27, 2011; posted June 27, 2011 (Doc. ID 146416); published July 21, 2011

We demonstrate third-harmonic generation (THG) in a dispersion-engineered slow-light photonic crystal waveguide fabricated in AMTIR-1 chalcogenide glass. Owing to the relatively low loss and low dispersion in the slowlight $(c / 30)$ regime, combined with the high nonlinear figure of merit of the material ( 2), we obtain a relatively large conversion efficiency $\left(1.4 \times 10^{-8} / \mathrm{W}^{2}\right)$, which is $30 \times$ higher than in comparable silicon waveguides, and observe a uniform visible light pattern along the waveguide. These results widen the number of applications underpinned by THG in slow-light platforms, such as the direct observation of the spatial evolution of the propagating mode. () 2011 Optical Society of America

OCIS codes: $130.5296,190.4160$.

Slow light in photonic crystal (PhC) waveguides has attracted significant attention for enhancing the efficiency of nonlinear optical processes over short path lengths [1-8]. The possibility of engineering the dispersion of slow-light modes in these structures $[2,3,6,8]$ has enabled the realization of broadband nonlinear devices that can operate at ultrahigh bit rates, with a record of $640 \mathrm{Gbit} / \mathrm{s}$ having been reported [9]. However, most of these demonstrations have used III-V $[4,5]$ and silicon semiconductors $[2,3,6-9]$ that are compromised by nonlinear losses induced both by two-photon absorption (TPA) and photogenerated free carriers. PhC waveguides have been recently fabricated in alternative materials with a higher nonlinear figure of merit (FOM)-FOM = $n_{2} / \lambda \beta_{\text {TPA }}\left(n_{2}\right.$ and $\beta_{\text {TPA }}$ are the Kerr and TPA coefficients, respectively) - such as wide bandgap III-V semiconductors $[10,11]$ or chalcogenide glasses (ChGs) [12-14]. However, so far, most of the associated nonlinear demonstrations have relied on band-edge slow light, which typically suffers from high loss and dispersion. The fabrication of $\mathrm{PhC}$ in less mature ChGs that have a lower refractive index relative to $\mathrm{Si}$, for example, remains challenging. Silver-doped $\mathrm{ChG} \mathrm{PhC}$ waveguides have been realized but exhibit a propagation loss of $\sim 170 \mathrm{~dB} / \mathrm{cm}$ [13], i.e., between 1 and 2 orders of magnitude larger than in silicon $\mathrm{PhC}$ waveguides [15]. A state-of-the-art approach for producing high-quality dispersion-engineered $\mathrm{PhC}$ waveguides in ChG with a FOM $>1$ has been developed only very recently [16].

Third-harmonic generation (THG) is a nonlinear optical process that manifests itself as the generation of visible light via the conversion of near-infrared (NIR) photons. This effect can be enhanced by slow-light propagation in $\mathrm{PhC}$ waveguides [7], with the periodic lattice also providing a way to extract the third-harmonic (TH) light out of a material that is, like $\mathrm{Si}$, opaque in the visible [17]. Although $\mathrm{PhC}$ microcavities have also been used successfully to enhance the efficiency of harmonic generation $[\underline{18}, 19]$, only the slow-light approach is compatible with wide bandwidth signals, thereby allowing the use of THG for high-speed all-optical signal processing [9], and supporting high-power ultrashort pulses yielding better conversion efficiencies. In this context, however, nonlinear losses not only restrict the THG conversion efficiency, but also the number of applications, such as in ultrahigh-bandwidth signal monitoring [9]. For example, the visible light emitted through THG in silicon tends to decay rapidly along the $\mathrm{PhC}$ waveguide over a short $(<40 \mu \mathrm{m})$ propagation distance, predominantly due to silicon's high nonlinear loss. Using a material with a higher nonlinear FOM should overcome this limitation.

Here, we demonstrate THG in dispersion-engineered slow-light PhC waveguides fabricated from AMTIR-1 ChG. The reasonably low linear propagation loss and suitably engineered dispersion, together with the high nonlinear FOM of the material (FOM $\sim 2$ [20]) yield an improved THG conversion efficiency compared to that measured in similar silicon structures $(\mathrm{FOM} \sim 0.3-0.5$ at $1550 \mathrm{~nm}$ ). Most importantly, the pattern of visible light generated along the $100-\mu \mathrm{m}$-long waveguide can be quite uniform, indicating that the TH conversion process is not limited by linear or nonlinear losses.

Our sample consists of a $100-\mu$ m-long AMTIR-1 $\left(\mathrm{Ge}_{33} \mathrm{As}_{12} \mathrm{Se}_{55}\right)$ ChG PhC waveguide suspended in air and connected to tapered ChG ridge waveguides on both sides to improve light insertion in a butt-coupling configuration. The chip was fabricated using $e$-beam lithography; chemically assisted ion beam etching; and vapor phase hydrofluoric acid etching from a 300-nm-thick AMTIR-1 film (refractive index $n \sim 2.6$ ) evaporated onto $\mathrm{a} \mathrm{SiO}_{2} / \mathrm{Si}$ wafer [16]. The $\mathrm{PhC}$ waveguide was created by omitting one row of holes in the $\Gamma K$ direction of the triangular PhC lattice (period a $\sim 525 \mathrm{~nm}$ and hole radius $\sim 0.3 a$ ), and the first two rows of holes around the defect were laterally shifted to engineer the dispersion of the 


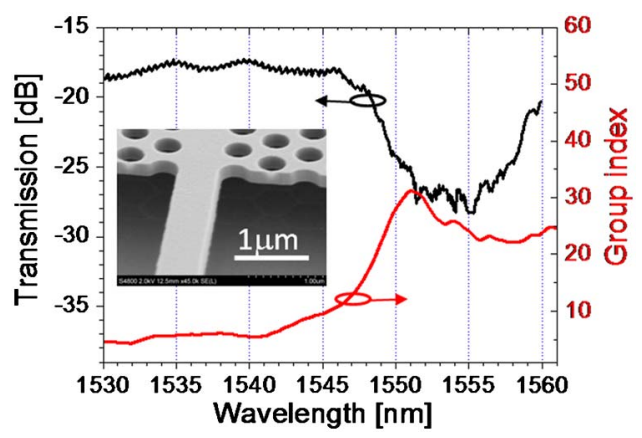

Fig. 1. (Color online) Transmission and measured group index dispersion of the waveguide in the NIR. Inset: scanning electron microscope picture.

fundamental mode. Ref [16] contains further details on the PhC waveguide geometry and its fabrication. Figure 1 shows the measured group index dispersion of the resulting $\mathrm{PhC}$ waveguide. This curve displays a flatband slow-light window centered on $1555 \mathrm{~nm}$ exhibiting a group velocity of $\sim c / 28 \pm 10 \%$ over $\sim 10 \mathrm{~nm}$. The secondorder dispersion parameter $\beta_{2}$ is at most equal to $\sim 5 \times$ $10^{-21} \mathrm{~s}^{2} / \mathrm{m}$ in this band. On the same plot, the transmission of the waveguide has been superimposed. It exhibits a dip around $\sim 1555 \mathrm{~nm}$, whose origin may be related to a variation of the propagation loss across the slow-light band, as discussed below.

We probed the waveguide by launching $1.2 \mathrm{ps}$ pulses (4 $\mathrm{MHz}$ repetition rate) generated by a mode-locked fiber laser at a wavelength tunable around $1550 \mathrm{~nm}$. After amplification, the NIR pump signal was coupled into the TE mode of the waveguide using lensed fibers (see [2,7]), with an estimated efficiency of $\sim 14 \%$. Figure 2 shows the transmission of the NIR light when increasing the coupled peak power up to $\sim 5 \mathrm{~W}(200 \mu \mathrm{W}$ average input power) at $1559 \mathrm{~nm}$. No significant saturation of the transmission was observed in this power range, confirming the relatively high nonlinear FOM of AMTIR-1 and the absence of nonlinearly generated free carriers in this glass. There is still, however, a roll-off above $\sim 3 \mathrm{~W}$, from which a $\beta_{\text {TPA }}$ coefficient of $\sim 4 \times 10^{-10} \mathrm{~m} / \mathrm{W} \pm 25 \%$ is inferred at $1559 \mathrm{~nm}\left(n_{g} \sim 25\right)$ and $\sim 9 \times 10^{-11} \mathrm{~m} / \mathrm{W} \pm 25 \%$ at $1545 \mathrm{~nm}$ $\left(n_{g} \sim 10\right)$ [5]. These values are reasonably consistent with the nominal AMTIR- $1 \beta_{\text {TPA }}$ of $4 \times 10^{-12} \mathrm{~m} / \mathrm{W} \pm 25 \%$

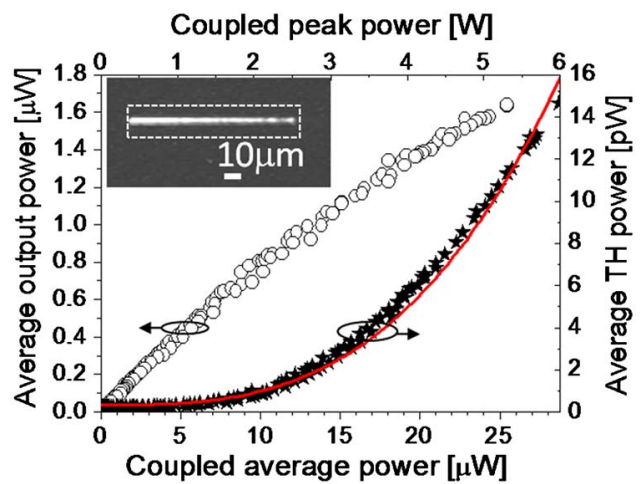

Fig. 2. (Color online) (Left) NIR light transmission versus pump power at $1559 \mathrm{~nm}$. (Right) TH light average power versus pump power at $1559 \mathrm{~nm}$ (stars) along with a cubic fit (red curve). Inset: visible image collected from the top of the PhC waveguide with a CCD camera (NIR signal launched left to right). measured in the NIR [20], when factoring out the scaling of this parameter with the square of the group index [2].

Because of the optical Kerr response of ChG, a fraction of the NIR pump is converted into TH visible light. The inset of Fig. 2 shows an image recorded by a CCD camera after collection of the TH light at $\sim 520 \mathrm{~nm}$ from the top of the waveguide using an N.A. $=0.42$ objective. The pattern of visible light is relatively uniform along the waveguide, in contrast with what has been observed in $\mathrm{Si} \mathrm{PhC}$ waveguides [7]. We measured the average power of $\mathrm{TH}$ visible light $\left(P_{3 \omega}\right)$ with a sensitive $\mathrm{Si}$ photodiode as a function of the pump power $\left(P_{\omega}\right)$. Figure 2 shows the resulting curve along with a cubic fit (red line) expected for the THG process $\left(P_{3 \omega} \propto P_{\omega}^{3}\right)$. No major deviation from the cubic fit was observed, reemphasizing the negligible contribution of nonlinear loss for the power range considered. The measured THG conversion efficiency $\eta=P_{3 \omega} / P_{\omega}$ was $\sim 4 \times 10^{-7}$ at $5 \mathrm{~W}$ peak power and $1.4 \times$ $10^{-8}$ for $1 \mathrm{~W}$ peak power, which is $\sim 30 \times$ times larger than that in similar slow-light waveguides made in $\mathrm{Si}$ [7]. This improvement is roughly consistent with the $\sim 3 \times$ larger nonlinear index $n_{2}$ of AMTIR-1 at $1550 \mathrm{~nm}$ [20] relative to that of $\mathrm{Si}\left(\eta\right.$ scales as $n_{2}^{2}$ [7]) and its lower nonlinear loss. This represents a lower bound value for the actual conversion efficiency, due to the limited TH light extraction efficiency. The latter is predominantly restricted by the massive AMTIR-1 absorption of the TH light, greater than $17 \mathrm{~dB} / \mu \mathrm{m}$ below the material bandgap at $600 \mathrm{~nm}$; but higher efficiencies could be achieved with lower group velocity modes.

Because of the strong absorption of $\mathrm{ChG}$ in the visible, the TH light profile along the waveguide axis directly reflects the intensity variations of the NIR signal (due to loss and dispersion) as it propagates across the waveguide, similarly to what was demonstrated in $\mathrm{Si}$ [17]. Because the TH power depends cubically on the pump power, these variations are enhanced in observations of the TH light distribution. Considering the value of the dispersion length $\left(L_{D}>300 \mu \mathrm{m}\right.$ for $1.2 \mathrm{ps}$ NIR pulses), which is much larger than the physical length of the waveguide, and the negligible contribution from the nonlinear loss in ChG, the main factor affecting the fundamental mode intensity variation along the waveguide here is the propagation loss. Figure 3(a) displays a series of CCD images showing the TH visible light when tuning the pump wavelength across and slightly below the flatband slow-light window.

In addition to the overall $\mathrm{TH}$ power variation due to the group velocity changes of the NIR mode across the probed spectral band, the TH visible light pattern changes significantly. It is relatively uniform toward short $(<1548 \mathrm{~nm})$ and long $(>1558 \mathrm{~nm})$ wavelengths, but it decays more rapidly along the waveguide axis in between. These intermediate wavelengths roughly coincide with the dip in transmission observed in Fig. 1. From these images, an estimate of the TH decay length was extracted and compared with the effective length inferred from the NIR pulsed signal low-power transmission as in Fig. 2 at different wavelengths (assuming a constant coupling efficiency into the PhC across 1540-1560 nm). A relatively good agreement was obtained, despite a $\sim 3 \mathrm{~nm}$ wavelength shift due to some photosensitivity of AMTIR-1 [21] between the two series of measurements. 
(a)
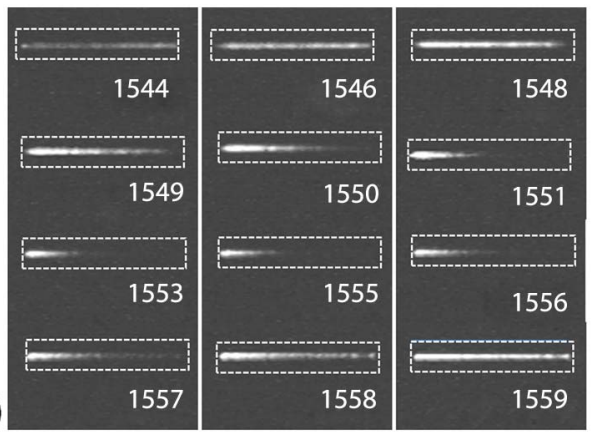

(b)

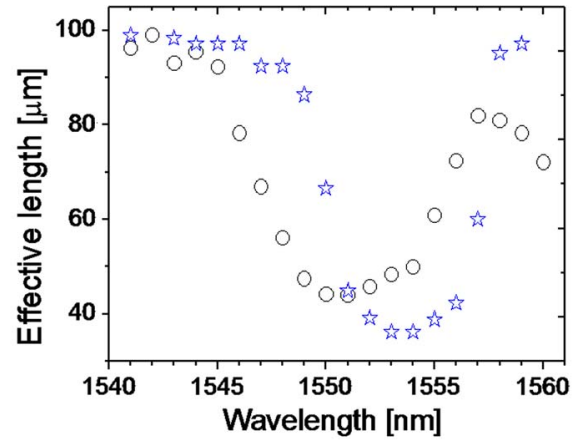

Fig. 3. (Color online) (a) TH visible light images at various pump wavelengths, as indicated. The dotted rectangle highlights the boundary of the $\mathrm{PhC}$, with the pump launched from left to right. (b) Effective length versus pump wavelength, inferred from the NIR low power pulse transmission as in Fig. 2 (circles) and from the decay length of the TH profile (stars).

The TH light therefore allows us to qualitatively estimate the variation of the propagation loss for the slow-light waveguide. The analysis of the TH Fourier space images (as in Ref [17]) excludes the role of multimode propagation as a possible cause for this transmission dip. It likely originates from a different scaling of the extrinsic out-ofplane and backscattering losses with $v_{g}$ [22], as modified with respect to $\mathrm{Si}$ structures by the $\mathrm{Ch} \overline{\mathrm{G}}$ lower refractive index.

In conclusion, we have reported THG in dispersionengineered slow-light $\mathrm{PhC}$ waveguides, fabricated in a ChG with a nonlinear FOM $>1$ and no free carrier effects. The results and their comparison with that obtained in $\mathrm{Si}$ structures show the benefits that can be gained from using a nonlinear material with a higher nonlinear FOM. Not only is the conversion efficiency larger $\left(1.4 \times 10^{-8} / \mathrm{W}^{2}\right)$, a 30 -fold improvement compared to silicon, but the spatial pattern of TH light is also more uniform along the waveguide. Because the process is much less degraded by nonlinear loss, the TH visible light distribution in this platform gives direct information about the NIR propagation loss of the PhC waveguide, providing, in principle, a sensitive tool to qualitatively study the scaling of the propagation losses with $v_{g}$.

This work was supported by the Centre of Excellence and Federation Fellowship programs of the Australian Research Council, as well as the School of Physics and the University of Sydney. We also acknowledge the
European Union and the UK Engineering and Physical Sciences Research Council through the funding of the Slow Photon Light Activated Switch (SPLASH) project and the Silicon Photonics project.

\section{References}

1. T. Baba, Nat. Photonics 2, 465 (2008).

2. C. Monat, B. Corcoran, M. Ebnali-Heidari, C. Grillet, B. J. Eggleton, T. P. White, L. O'Faolain, and T. F. Krauss, Opt. Express 17, 2944 (2009).

3. Y. Hamachi, S. Kubo, and T. Baba, Opt. Lett. 34, 1072 (2009).

4. A. Baron, A. Ryasnyanskiy, N. Dubreuil, P. Delaye, Q. Vy Tran, S. Combrié, A. de Rossi, R. Frey, and G. Roosen, Opt. Express 17, 552 (2009).

5. K. Inoue, H. Oda, N. Ikeda, and K. Asakawa, Opt. Express 17, 7206 (2009).

6. C. Monat, B. Corcoran, D. Pudo, M. Ebnali-Heidari, C. Grillet, M. D. Pelusi, D. J. Moss, B. J. Eggleton, T. P. White, L. O'Faolain, and T. F. Krauss, IEEE J. Sel. Top. Quantum Electron. 16, 344 (2010).

7. B. Corcoran, C. Monat, C. Grillet, D. J. Moss, B. J. Eggleton, T. P. White, L. O'Faolain, and T. F. Krauss, Nat. Photonics 3. 206 (2009).

8. C. Monat, M. Ebnali-Heidari, C. Grillet, B. Corcoran, B. J. Eggleton, T. P. White, L. O'Faolain, J. Li, and T. F. Krauss, Opt. Express 18, 22915 (2010).

9. B. Corcoran, C. Monat, M. Pelusi, C. Grillet, T. P. White, L. O'Faolain, T. F. Krauss, B. J. Eggleton, and D. J. Moss, Opt. Express 18, 7770 (2010).

10. S. Combrie, Q. V. Tran, A. De Rossi, C. Husko, and P. Colman, Appl. Phys. Lett. 95, 221108 (2009).

11. P. Colman, C. Husko, S. Combrie, I. Sagnes, C. W. Wong, and A. De Rossi, Nat. Photonics 4, 862 (2010).

12. M. W. Lee, C. Grillet, C. G. Poulton, C. Monat, C. L. C. Smith, E. Mägi, D. Freeman, S. Madden, B. Luther-Davies, and B. J. Eggleton, Opt. Express 16, 13800 (2008).

13. K. Suzuki, Y. Hamachi, and T. Baba, Opt. Express 17, 22393 (2009).

14. B. J. Eggleton, B. Luther-Davies, and K. Richardson, Nat. Photonics 5, 141 (2011).

15. L. O'Faolain, X. Yuan, D. McIntyre, S. Thoms, H. Chong, R. M. De la Rue, and T. F. Krauss, Electron. Lett. 42, 1454 (2006).

16. M. Spurny, L. O'Faolain, D. A. P. Bulla, B. Luther-Davies, and T. F. Krauss, Opt. Express 19, 1991 (2011).

17. C. Monat, C. Grillet, B. Corcoran, D. J. Moss, B. J. Eggleton, T. P. White, and T. F. Krauss, Opt. Express 18, 6831 (2010).

18. K. Rivoire, Z. L. Lin, F. Hatami, W. T. Masselink, and J. Vuckovic, Opt. Express 17, 22609 (2009).

19. M. Galli, D. Gerace, K. Welna, T. F. Krauss, L. O'Faolain, G. Guizzetti, and L. C. Andreani, Opt. Express 18, 26613 (2010).

20. J. T. Gopinath, M. Soljacic, E. P. Ippen, V. N. Fuflyigin, W. A. King, and M. Shurgalin, J. Appl. Phys. 96, 6931 (2004).

21. M. W. Lee, C. Grillet, C. Monat, E. Magi, S. TomljenovicHanic, X. Gai, S. Madden, D. Y. Choi, D. Bulla, B. LutherDavies, and B. J. Eggleton, Opt. Express 18, 26695 (2010).

22. L. O'Faolain, S. A. Schulz, D. M. Beggs, T. P. White, M. Spasenović, L. Kuipers, F. Morichetti, A. Melloni, S. Mazoyer, J. P. Hugonin, P. Lalanne, and T. F. Krauss, Opt. Express 18, 27627 (2010). 